\title{
Population Genetic Difference of Pharmacogenomic VIP Variants in the Tibetan Population
}

\author{
Chunjuan $\mathrm{He}^{\prime}$ \\ Linna Peng' \\ Shishi Xing \\ Dandan $\mathrm{Li}^{\prime}$ \\ Li Wang (iD) \\ Tianbo Jin (1) ${ }^{1,2}$ \\ 'Key Laboratory of Molecular Mechanism \\ and Intervention Research for Plateau \\ Diseases of Tibet Autonomous Region, \\ School of Medicine, Xizang Minzu \\ University, Xianyang, Shaanxi, 7/2082, \\ People's Republic of China; ${ }^{2}$ Key \\ Laboratory for Basic Life Science \\ Research of Tibet Autonomous Region, \\ Xizang Minzu University, Xianyang, \\ Shaanxi, 712082, People's Republic of \\ China
}

Background: Genetic variation influences drug reaction or adverse prognosis. The purpose of this research was to genotype very important pharmacogenetic (VIP) variants in the Tibetan population.

Methods and Materials: Blood samples from 200 Tibetans were randomly collected and 59 VIP variants were genotyped, and then compared our data to 26 other populations in the 1000 project to further analyze and identify significant difference.

Results: The results showed that on comparing with most of the 26 populations from the 1000 project, rs4291 (ACE), rs1051296 (SLC19A1) and rs1065852 (CYP2D6) significantly differed in the Tibetan population. Furthermore, three significant loci were related to drug response. In addition, the allele frequency of Tibetans least differed from that of East Asian populations, and most differed from that of Americans.

Conclusion: Three significant loci of variation ACE rs4291, SLC19A1 rs1051296 and $C Y P 2 D 6$ rs1065852 were associated with drug response. This result will contribute to improving the information of the Tibetan in the pharmacogenomics database, and providing a theoretical basis for clinical individualised drug use in Tibetans.

Keywords: the Tibetan population, pharmacogenomics, VIP variants

\section{Introduction}

A major challenge in current drug clinical practice, drug development and drug regulation is the huge difference in drug treatment ${ }^{1}$ which is reasoned to the individual differences for the same drug. ${ }^{2}$ Single nucleotide polymorphism (SNP) is an important determinant of individual differences in drug therapy. Pharmacogenomics mainly involves knowledge of pharmacokinetic, pharmacodynamic and focuses on the inheritance of individual variation in the course of drug treatment, clarifies the influence of genomic variation on drug distribution and function, and provides guidance for individualised precise medical treatment ${ }^{3,4}$ so on. The Pharmacogenomics Knowledge Base (PharmGKB: http://www.pharmgkb.org) is a useful resource that aims to explain the gene-drug-disease relationship. In recent years, pharmacogenomics research has largely focused on genetic variations that may be related to drug response or metabolism. ${ }^{5}$ These variations-very important genetic (VIP) variants are mainly concentrated in the pharmacogenomics database. They assessed the relationship between the VIP variants and specific drugs, and provide individuals with appropriate drugs at proper doses. ${ }^{6}$

There are many differences in genetic heterogeneity and genetic polymorphism among different ethnic groups. ${ }^{7}$ An important research hotspot in pharmacogenomics 
is to compare the efficacy of drugs among different races. Tibetan is one of the largest of the 56 ethnic Chinese nation, with a long history. Most Tibetans reside in the Tibet Autonomous Region on the Qinghai-Tibet Plateau, and some people also live in Qinghai, Gansu, Sichuan, Yunnan and other regions of China. The Tibetans have gradually formed unique ethnic custom, lifestyles and eating habits based on the climate and altitude of their residing areas. A study of the genomic variants in the Tibetan population showed that most of the Tibetan gene pool may be differ from the gene pool of the Han people. ${ }^{8}$

This study aimed to assess the genotype frequencies of 59 VIP variants between Tibetans and the other 26 ethnicities from the 1000 genome project. The results will contribute to improving the information of the Tibetan pharmacogenomics database, and providing a theoretical basis for drug management in Tibetans.

\section{Materials and Methods Study Participants}

200 unrelated Tibetans were recruited from the Tibet Autonomous Region. According to the paternal lineage of at least past three generations, all candidates were confirmed to be Tibetan. Based on the results of physical examination, people with chronic diseases were excluded. The protocol of this study was known to all participants and they have signed informed consent. According to the ethics committee of Xizang Minzu University (2019-12), all participants provided blood samples. The procedures met the agency's ethical standards and the 1964 Helsinki Declaration and its subsequent amendments or similar ethical standards.

\section{Variant Selection, DNA Extraction and Genotyping}

The 59 VIP variants were selected from the PharmGKB database (http://www.pharmgkb.org). The genomic DNA of participants was extracted according to the extraction method of the blood DNA extraction kit (GoldMag Ltd. Xi'an, China), and then its DNA purity and concentration were inspected. Multiplexed SNP MassEXTEND assays were designed by the Agena MassARRAY Assay Design 3.0 software, and the Agena MassARRAY RS1000 was used to genotype the 59 VIP variants. ${ }^{9}$ Data arrangement and analysis was performed by Agena Typer 4.0 software. ${ }^{10,11}$

\section{Statistical Analyses}

To determine whether the genotype frequencies of variants were in Hardy-Weinberg equilibrium, we used Microsoft Excel and SPSS 22.0 (SPSS, Chicago, IL) for statistical analysis. The genotype frequencies of 59 variants in the Tibetan population were separately compared with those of the other super-populations downloaded from the 1000 genomes project (http://www.internationalgenome.org/), including (1)African: the African Caribbeans in Barbados (ACB); the African Ancestry in Southwest USA (ASW); Esan in Nigeria (ESN); Gambian in Western Divisions, The Gambia (GWD); the Luhya in Webuye, Kenya (LWK); the Mende in Sierra Leone (MSL); the Yoruba in Ibadan, Nigeria (YRI); (2)American: the Colombian in Medellin, Colombia (CLM); the Mexican Ancestry in Los Angeles, Colombia (MXL); the Peruvian in Lima, Peru (Peruvian in Lima, Peru); the Puerto Rican in Puerto Rico (PUR); (3)East Asian: the Chinese Dai in Xishuangbanna, China (CDX); the Han Chinese in Beijing, China (CHB0; the Southern Han Chinese, China (CHS); the Japanese in Tokyo, Japan (JPT); the Kinh in Ho Chi Minh City, Vietnam (KHV); (4)European: the Utah residents with Northern and Western European ancestry (CEU); the Finnish in Finland (FIN); the British in England and Scotland (GBR); the Iberian populations in Spain (IBS); the Toscani in Italy (TSI); (5)South Asian: the Bengali in Bangladesh (BEB); the Gujarati Indian in Houston, Texas (GIH); the Indian Telugu in the UK (ITU); the Punjabi in Lahore, Pakistan (PJL) and the Sri Lankan Tamil in the UK (STU). ${ }^{12}$ Also, Bonferroni's multiple adjustments were used for significance assessment, and all $p$ values were double-sided when $p<0.05$ and $p<0.05 /(59 \times 27)$, the $p$ value was considered to be statistically significant. ${ }^{13}$

\section{Results}

In the Table 1, we listed the basic characteristics of 59 VIP variants in 200 Tibetans and because of the nonspecific primers, 8 SNPs (rs1801252, rs1801160, rs2892949, rs12208357, rs1801279, rs10509681, rs1058930 and rs11572080) have been deleted. The selected SNP PCR primers were shown in Supplementary Table 1. The selected VIP gene information contained gene name, position, genotype frequency, the minor allele frequency and the functional consequence.

Based on the chi-squared test, as for the genotype frequencies of 59 variations, we made a comparison between Tibetans and other 26 nationalities (Supplementary Table 2). 


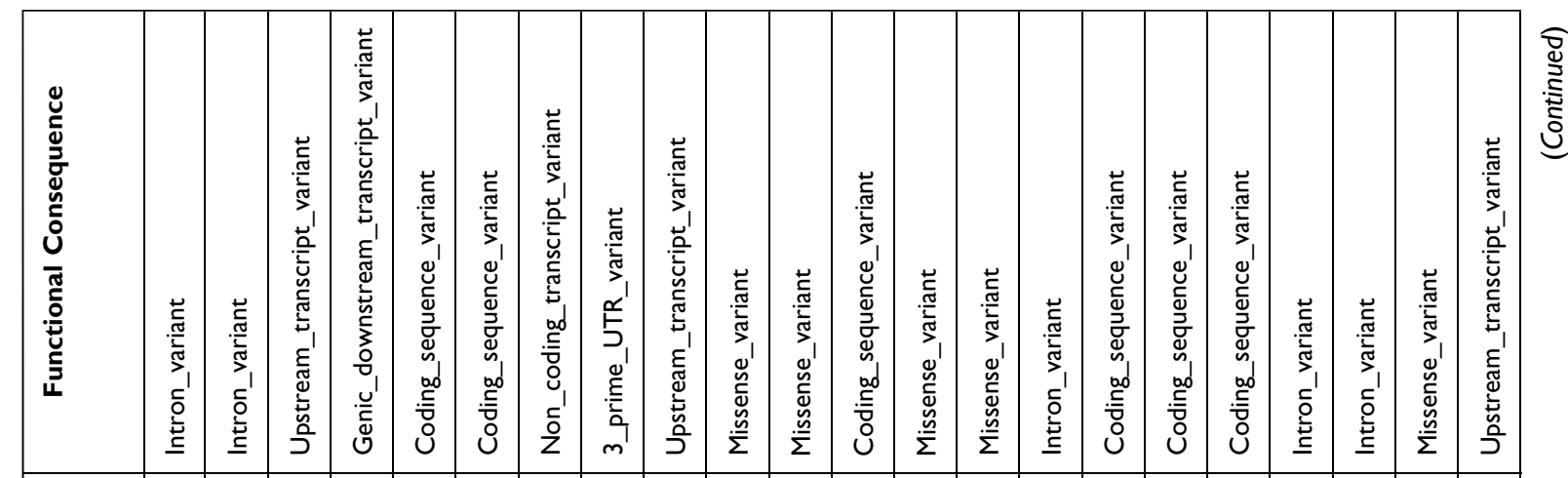

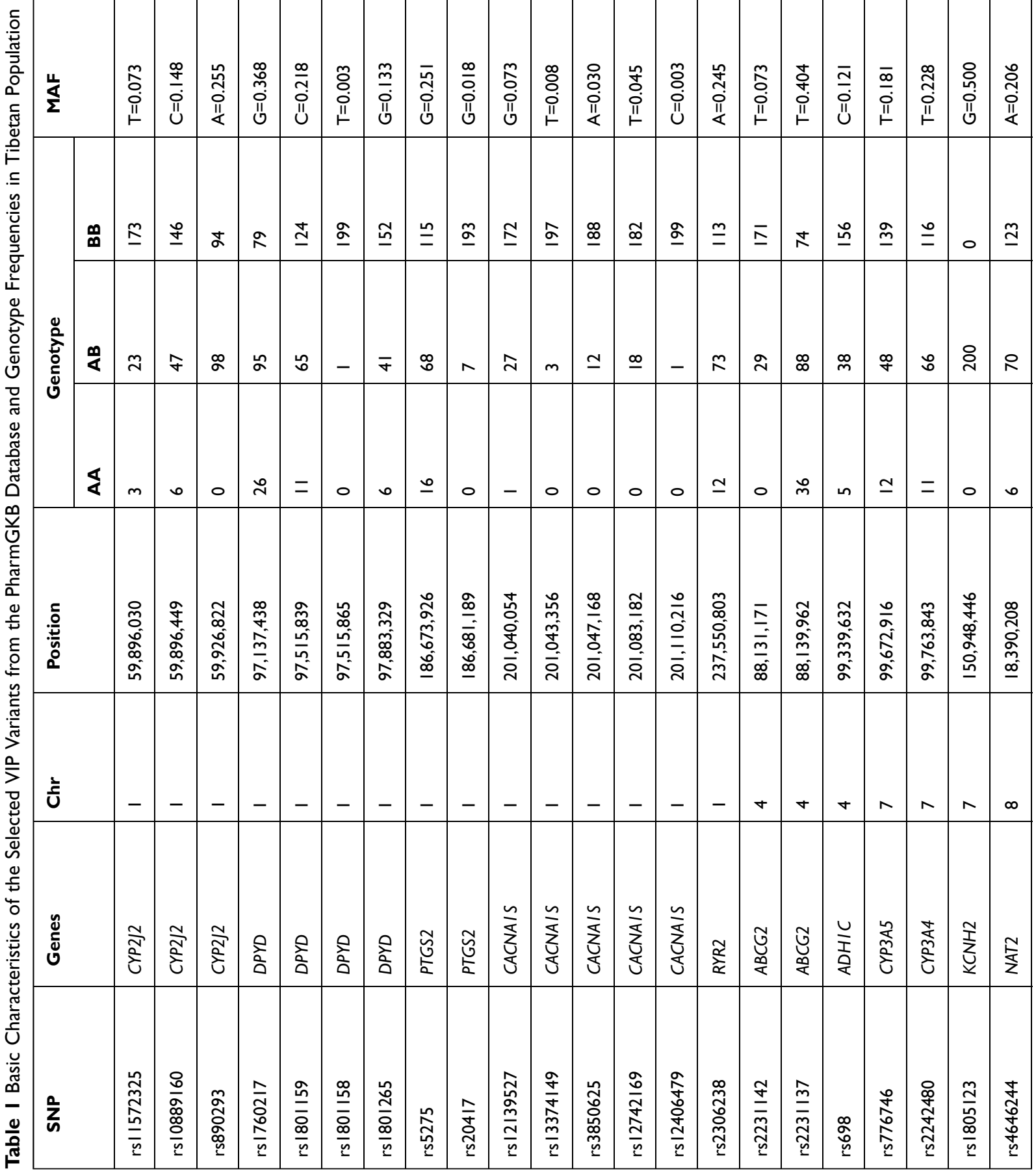




\begin{tabular}{|c|c|c|c|c|c|c|c|c|c|c|c|c|c|c|c|c|c|c|c|c|c|c|c|}
\hline 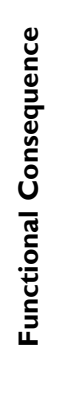 & 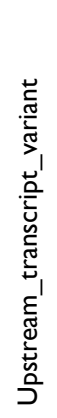 & 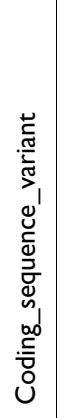 & 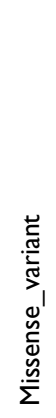 & 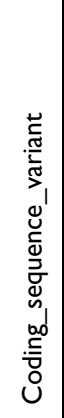 & 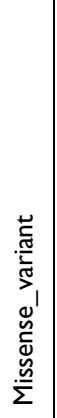 & 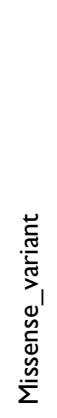 & 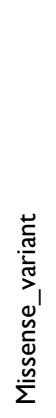 & $\begin{array}{l}0 \\
\text { ¿̇ }\end{array}$ & 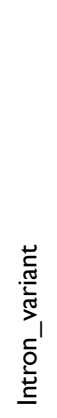 & 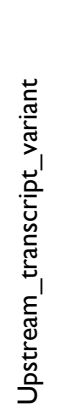 & 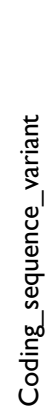 & 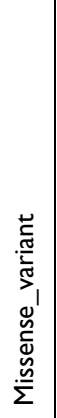 & 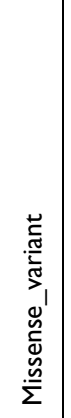 & 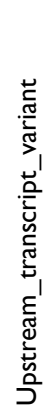 & 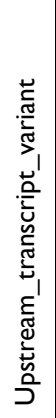 & 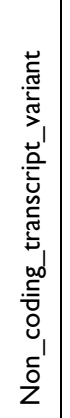 & 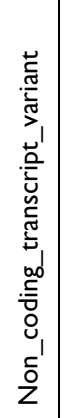 & 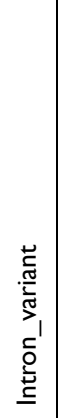 & 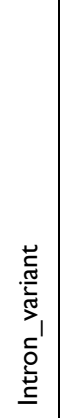 & 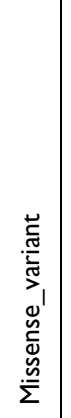 & 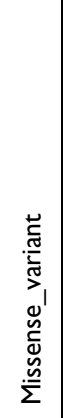 & 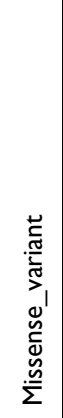 & 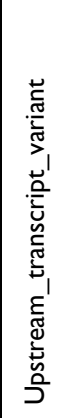 \\
\hline$\frac{u}{\Sigma}$ & $\begin{array}{l}\overline{\bar{n}} \\
\text { O⿱ } \\
\text { un }\end{array}$ & $\begin{array}{l}\overline{\bar{j}} \\
\text { II } \\
\qquad\end{array}$ & $\begin{array}{l}\text { o. } \\
\text { Ő } \\
\text { ü }\end{array}$ & 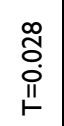 & 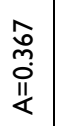 & 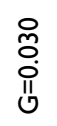 & $\frac{\stackrel{\infty}{\circ}}{\frac{0}{0}}$ & 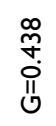 & $\frac{m}{\frac{m}{0}}$ & $\begin{array}{l}\text { n } \\
\overline{0} \\
\text { iI } \\
!\end{array}$ & 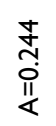 & 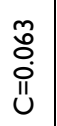 & $\begin{array}{l}\text { Oे } \\
\text { O̊ } \\
\text { II }\end{array}$ & $\frac{\Gamma}{\overline{0}}$ & $\begin{array}{l}\frac{0}{\pi !} \\
\stackrel{\Pi}{u}\end{array}$ & $\begin{array}{l}\text { L̊ } \\
\stackrel{0}{0} \\
\text { ü }\end{array}$ & $\begin{array}{l}\frac{\mathfrak{q}}{0} \\
\frac{\|}{\hbar}\end{array}$ & $\begin{array}{l}0 \\
\text { ò } \\
\text { Oा } \\
\text { L }\end{array}$ & $\frac{\stackrel{\sim}{\alpha}}{0}$ & $\begin{array}{l}\frac{\infty}{m} \\
\stackrel{0}{\Perp} \\
\vdash\end{array}$ & $\begin{array}{l}\infty \\
\stackrel{0}{0} \\
\stackrel{0}{0} \\
u\end{array}$ & $\underset{\substack{\text { N } \\
\text { II }}}{ }$ & $\begin{array}{l}\text { nू } \\
0 \\
0 \\
0 \\
u\end{array}$ \\
\hline
\end{tabular}

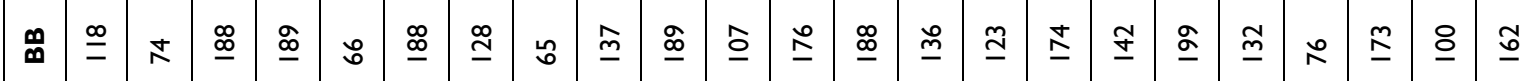

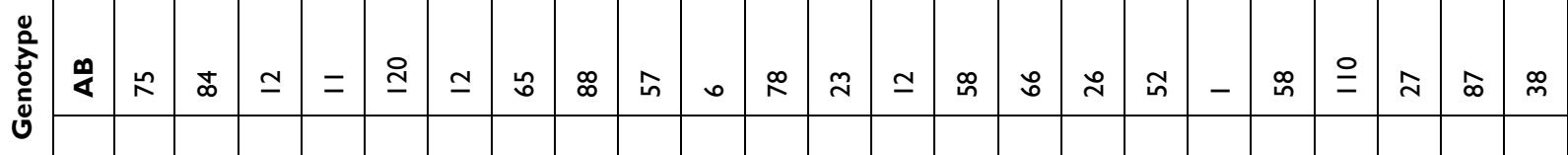

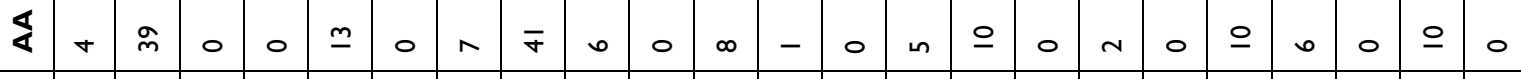

\begin{tabular}{|c|c|c|c|c|c|c|c|c|c|c|c|c|c|c|c|c|c|c|c|c|c|c|c|}
\hline 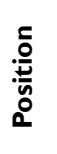 & $\begin{array}{l}0 \\
0 \\
0 \\
0 \\
0 \\
0\end{array}$ & 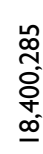 & $\begin{array}{l}\text { J } \\
\text { o } \\
\text { o } \\
\text { ó }\end{array}$ & 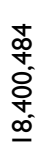 & 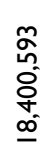 & $\begin{array}{l}0 \\
0 \\
0 \\
0 \\
0 \\
0\end{array}$ & 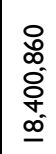 & 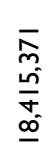 & 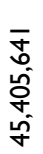 & $\begin{array}{l}\frac{2}{2} \\
\frac{1}{5} \\
\text { +2 }\end{array}$ & 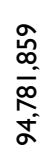 & 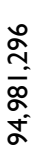 & 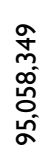 & 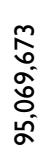 & 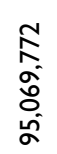 & 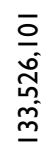 & 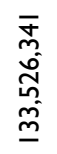 & 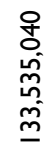 & 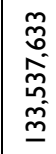 & 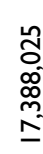 & 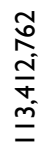 & 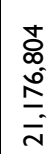 & \\
\hline
\end{tabular}

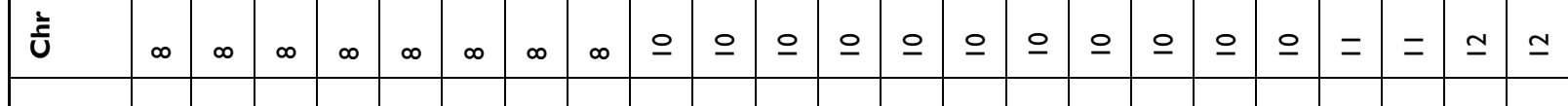

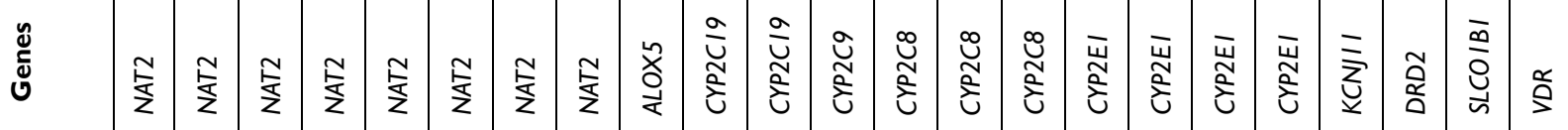

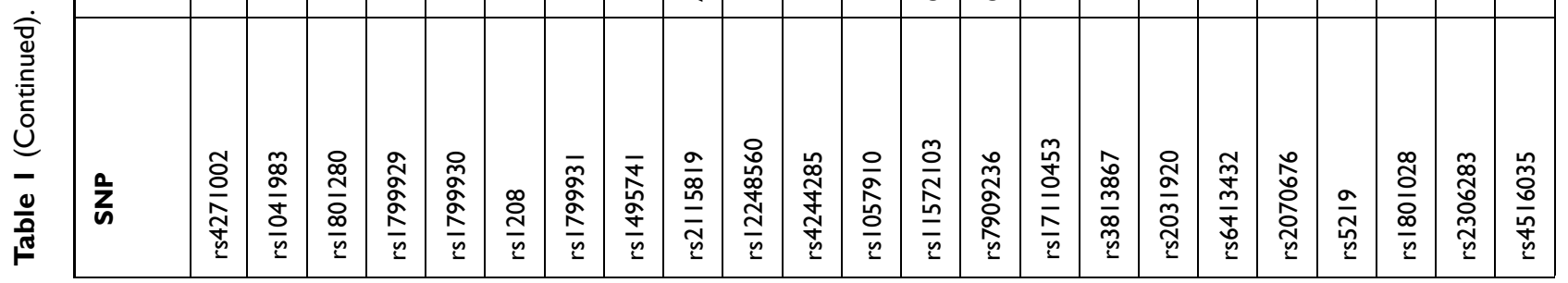




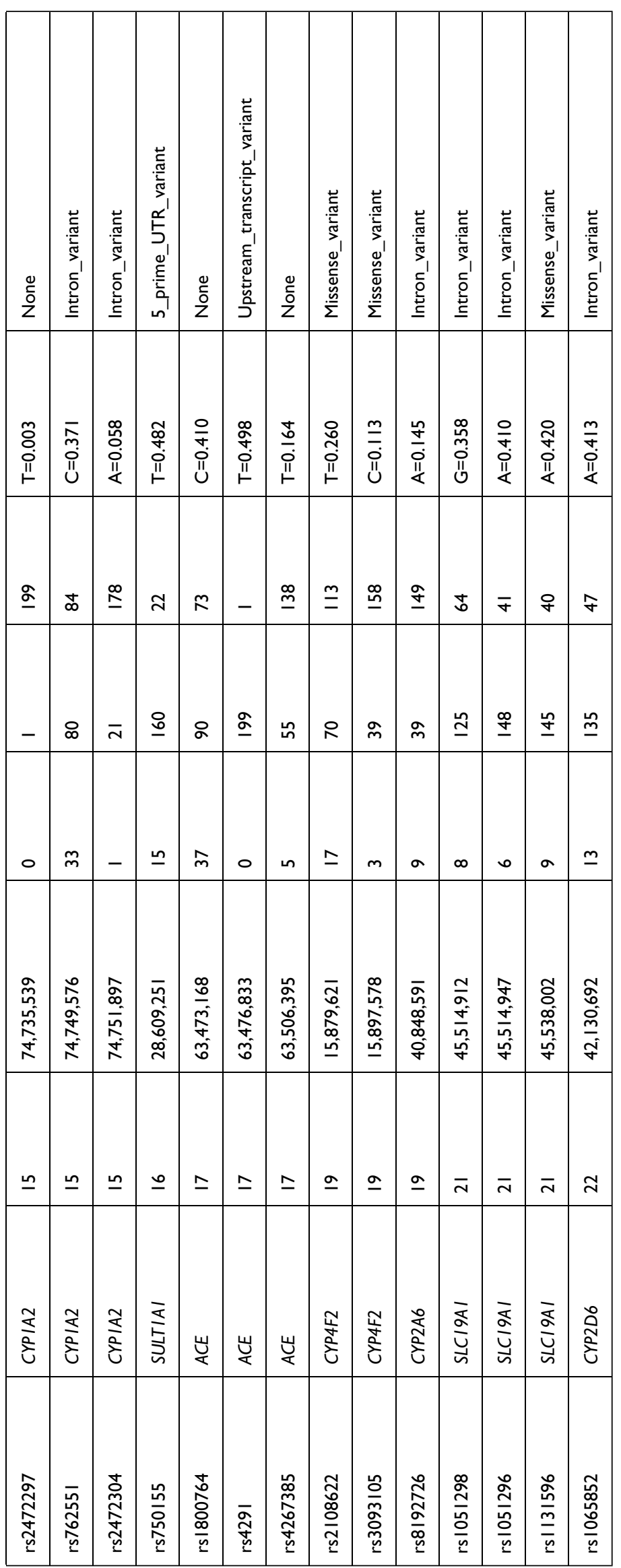


Before adjustments, $A C E$ rs4291, SLC19A1 rs1051296 and CYP2D6 rs1065852 significantly differed in the Tibetan population when compared with the other 26 nationalities. The SULT1A1 rs750155, SLC19A1 rs1051298 and SLC19A1 rs1131596 in the Tibetan population were obviously different from those of the other nationalities. Bonferroni adjustments have been made $(p<0.05 /(59 \times 27))$. The results showed that $A C E$ rs4291 and CYP2D6 rs1065852 were significantly different in the Tibetan population when compared to the other 26 nationalities. Except for CHS, SLC19A1 rs1051296 significantly differed in Tibetans when compared with the other 25 ethnic groups. Moreover, SULT1A1 rs750155 and SLC19A1 rs1131596 in the Tibetan population differed from those of the other 22 nationalities.

In addition, on the basis of Pharmgkb database (https:// www.pharmgkb.org/), Table 2 showed the drug-related information of significant difference. The rs4291 carriers with different genotypes have different responses to drugs (sertraline, captopril, aspirin and amlodipinechlorthalidonelisinoprilj). Rs1065852 was found to be related to alphahydroxymetoprolol, citalopram escitalopram and iloperidone. As for rs1051298, compared to the allele A individuals, individuals with allele $\mathrm{G}$ were associated with increased progression free survival after bevacizumab and pemetrexed treatment. Compared with allele $\mathrm{C}$, rs750155 T allele was not associated with ABT-751 pharmacokinetic parameters in cancer patients receiving ABT751 treatment. And when comparing to the allele A, rs1131596 $\mathrm{G}$ allele was not correlated with the response to methotrexate in children with precursor cell lymphoblastic leukaemia lymphoma and patients with rheumatoid arthritis. The correlation between rs1051296 and clinical drugs has not been reported to date.

The MAF distribution map of SNPs $A C E$ (rs4291 T allele), SLC19A1 (rs1051296 A allele), CYP2D6 (rs1065852 A allele), SULT1A1 (rs750155 T allele), SLC19A1 (rs1051298 G allele) and SLC19A1 (rs1131596 A allele) were compared with significant differences between the Tibetans and the other super-populations. As shown in Supplementary Figure 1, the allele frequency of the Tibetans least differed from the East Asian populations, and most differed from Americans. Among them, the frequencies of rs4291-T and rs1065852-A were the highest in the East Asian population, whereas the frequency of rs1051296-A was the highest in the African population. The frequency of rs1051298-G, rs750155-T and rs1131596-A were the highest in the American population.

\section{Discussion}

In recent years, many studies have focused on the efficacy comparison of drug reactions among different races, which lays a foundation for clinical individualised drug use. This study identified 59 VIP loci in Tibetans and compared them with the other 26 different populations. Three significant loci of variation were inferred from the genotyping results, $A C E$ rs4291, SLC19A1 rs1051296 and CYP2D6 rs1065852, which were associated with drug response.

Angiotensin-converting enzyme (ACE) encodes an enzyme involved in blood pressure regulation and electrolyte balance. It catalyses the conversion of angiotensin I into the physiologically active peptide angiotensin II. Angiotensin II promotes hypertension through vasoconstriction and salt and water retention, and is helpful for heart remodelling, inflammation, thrombosis and plaque rupture. ${ }^{14}$ At present, ACE inhibitors are the first-line treatment for hypertension, which can favourably affect the remodelling of patients with myocardial infarction and heart failure, and reduce the incidence rate and mortality. ${ }^{15}$ Therefore, ACE-encoded enzyme is the drug target. ${ }^{16,17}$ Polymorphic loci have been reported to play a role. ${ }^{18,19}$ Martínez-Rodríguez et $\mathrm{al}^{20}$ reported that rs4291 was associated with an increased risk of hypertension after adjusting for age, gender, BMI, triglyceride, drinking and smoking and with the elevation of ACE enzyme levels. In addition, the function of $A C E$ polymorphisms in Alzheimer's Disease cannot be ignored. The study by de Oliveira et $\mathrm{al}^{21}$ showed that ACE inhibitors, not related to blood pressure, can delay cognitive decline in ACE haplotype carriers with rs 1800764-T and rs4291-A. In 2016, they also observed that each A allele of rs4291 resulted in an increase of $3.074 \mathrm{mg} / \mathrm{dL}$ urea and 0.044 $\mathrm{mg} / \mathrm{dl}$ creatinine per year. The use of ACE inhibitors had a protective effect on the change of creatinine, and had no effect on the change of blood pressure. ${ }^{22}$ The above research showed that rs4291 carriers with different genotypes had different drug sensitivity.

The CYP2D6 gene encoded Cytochrome P450 Family 2 Subfamily D Member 6 that catalyses drug metabolism and the synthesis of cholesterol, steroids and other lipids. One report has shown that the gene is highly polymorphic in the population. Lee et $\mathrm{al}^{23}$ evaluated the association between CYP polymorphisms and the blood concentrations of hydroxychloroquine (HCQ) and its metabolite N-desethyl HCQ (DHCQ) in Korean patients with lupus. They observed that patients with CYP2D6*10 (rs1065852) GG genotype had the highest [DHCQ]/[HCQ] ratio, while patients with AA genotype had the lowest [DHCQ]/[HCQ] 


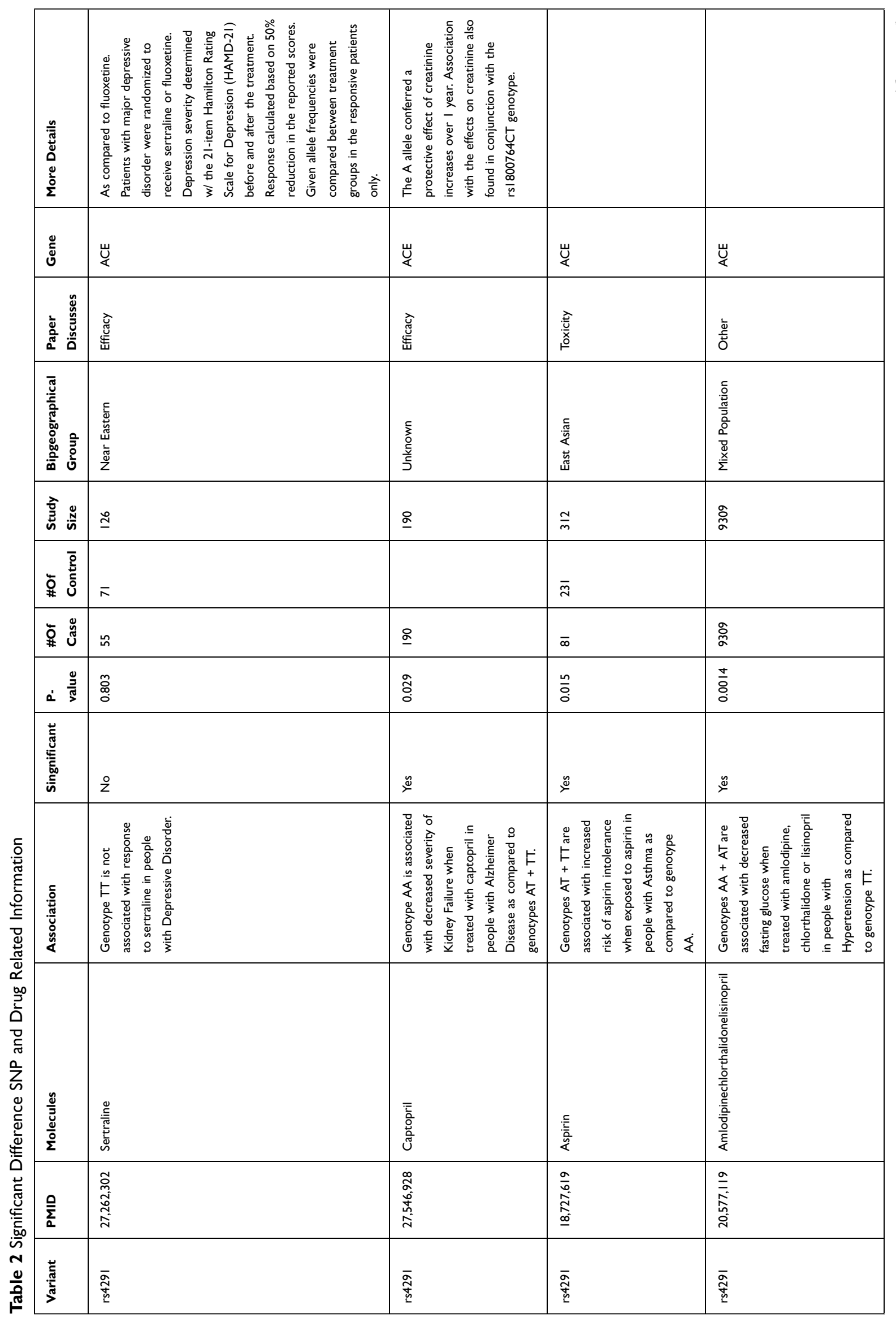




\begin{tabular}{|c|c|c|c|}
\hline 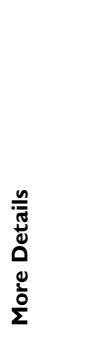 & 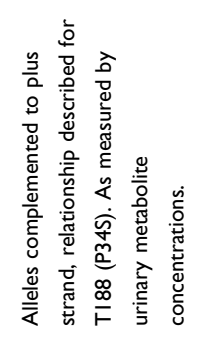 & 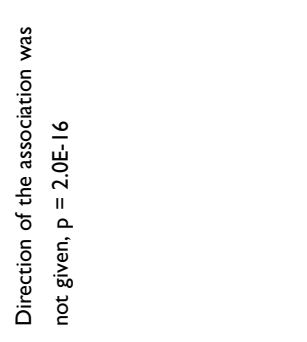 & 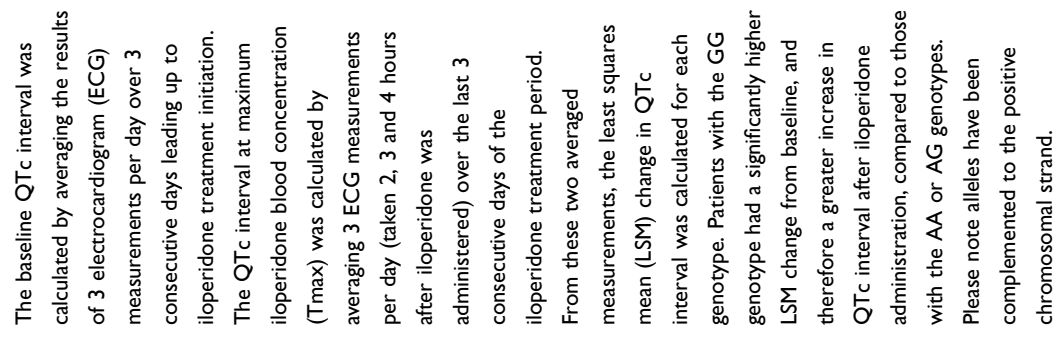 \\
\hline סّ̊ & 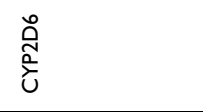 & 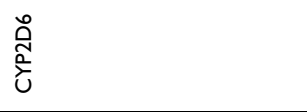 & 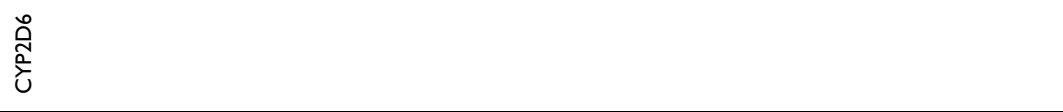 \\
\hline 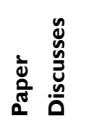 & 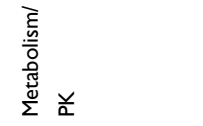 & 离 & $\begin{array}{l}\text { 离 } \\
\text { Oे }\end{array}$ \\
\hline 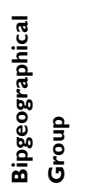 & 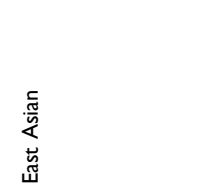 & 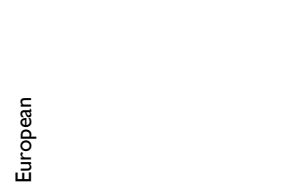 & $\begin{array}{l}\frac{5}{5} \\
\frac{5}{5} \\
\frac{5}{5}\end{array}$ \\
\hline 离 & g & $\stackrel{\tilde{q}}{\sigma}$ & $\stackrel{\infty}{\simeq}$ \\
\hline 离 & & & \\
\hline 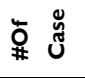 & o & $\stackrel{\stackrel{\sim}{q}}{\sigma}$ & $\stackrel{\infty}{\simeq}$ \\
\hline 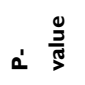 & $\begin{array}{l}\text { 㟔 } \\
\text { o } \\
\text { v }\end{array}$ & $\stackrel{\circ}{\stackrel{\sim}{\sim}}$ & 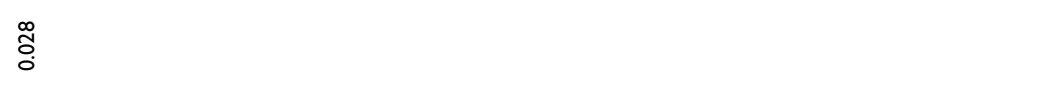 \\
\hline & $\stackrel{\stackrel{\varpi}{*}}{ }$ & $\stackrel{\square}{ٌ}$ & 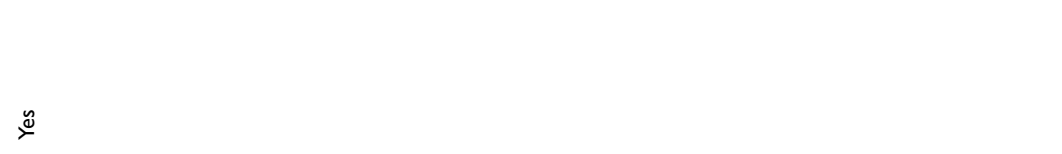 \\
\hline 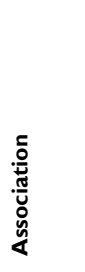 & 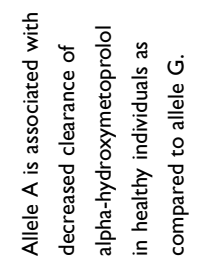 & 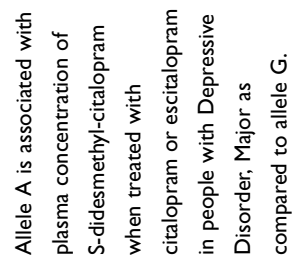 & 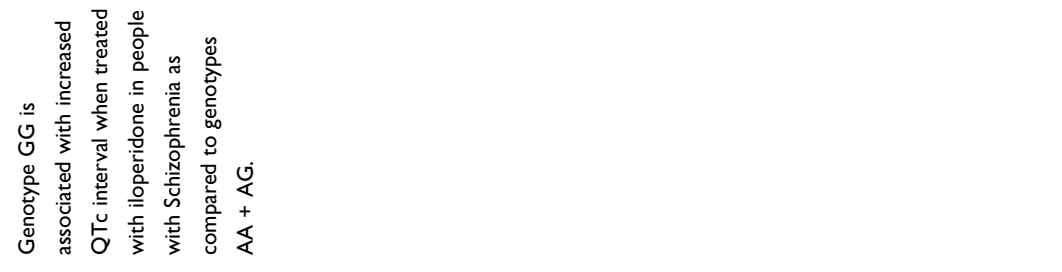 \\
\hline & 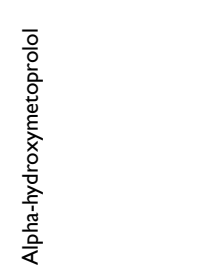 & 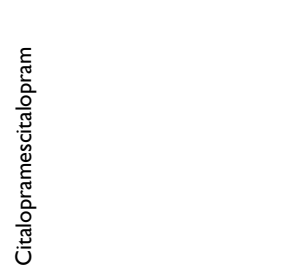 & 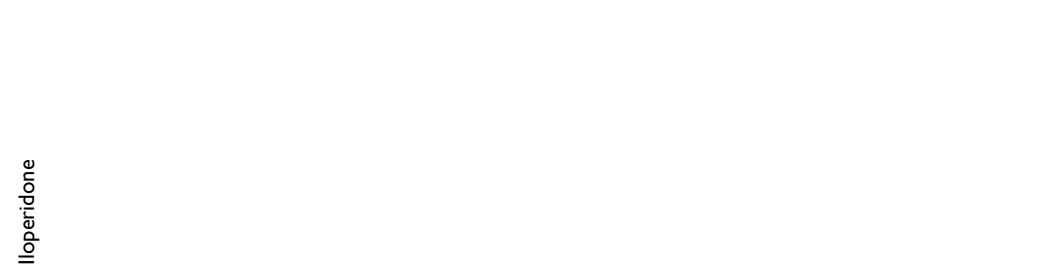 \\
\hline$\frac{\varrho}{\sum_{0}}$ & $\begin{array}{l}\hat{E} \\
\stackrel{\sim}{\pi} \\
\underline{\underline{\Xi}}\end{array}$ & 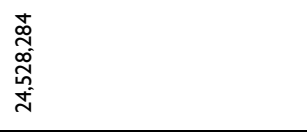 & 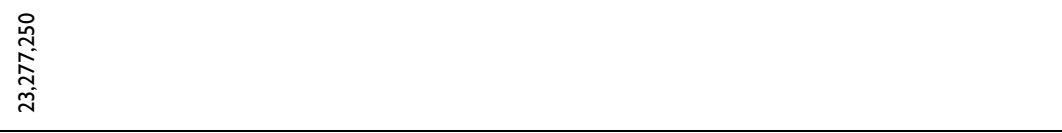 \\
\hline 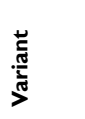 & 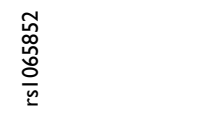 & 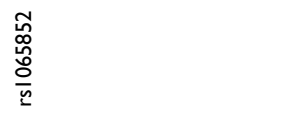 & 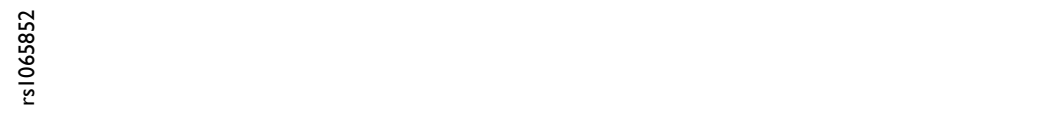 \\
\hline
\end{tabular}




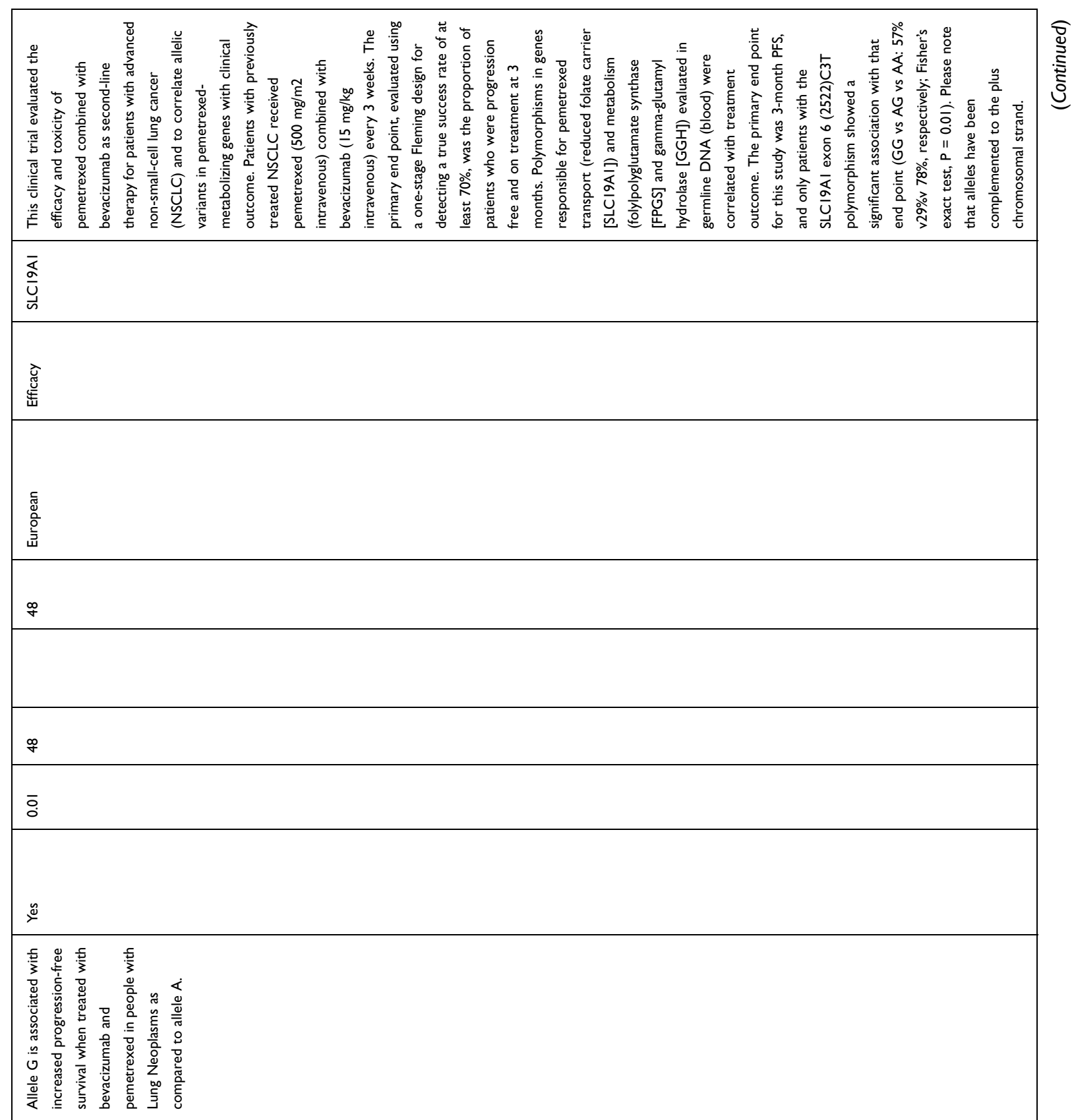

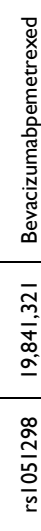




\begin{tabular}{|c|c|c|c|c|c|}
\hline 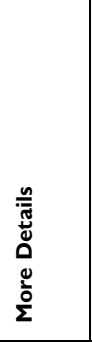 & 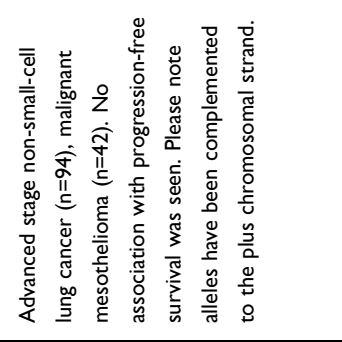 & 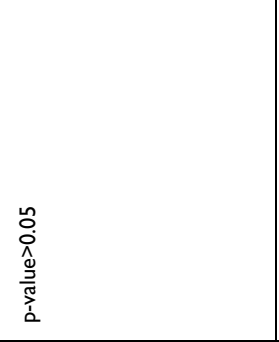 & 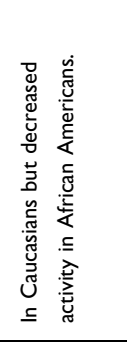 & 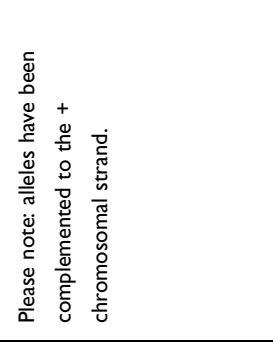 & 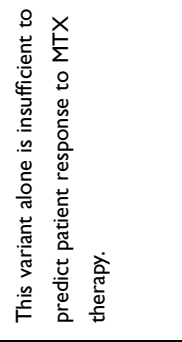 \\
\hline $\begin{array}{l}\stackrel{0}{\tilde{E}} \\
\stackrel{\tilde{e}}{0}\end{array}$ & $\begin{array}{l}\bar{\Xi} \\
\bar{U} \\
\bar{u}\end{array}$ & 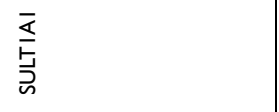 & $\begin{array}{l}\bar{s} \\
\stackrel{\Xi}{5} \\
心\end{array}$ & 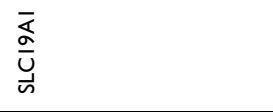 & $\begin{array}{l}\bar{\alpha} \\
\bar{u} \\
\bar{\omega}\end{array}$ \\
\hline 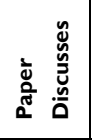 & 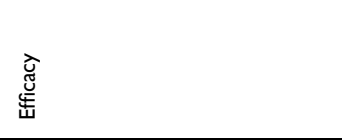 & 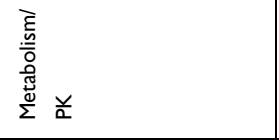 & & $\begin{array}{l}\text { 氙 } \\
\text { 密 }\end{array}$ & \\
\hline 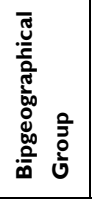 & 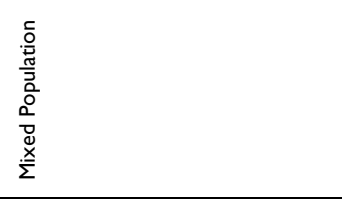 & 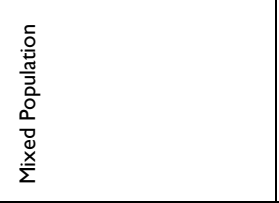 & & 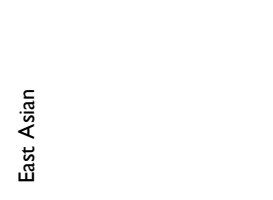 & $\begin{array}{l}\frac{5}{5} \\
\frac{0}{5} \\
\frac{5}{5}\end{array}$ \\
\hline 离 & $\stackrel{\circ}{\underline{m}}$ & $\hat{f}$ & & $\frac{\hat{m}}{m}$ & $\stackrel{\circ}{\circ}$ \\
\hline 总 & & & & & \\
\hline ¿ & 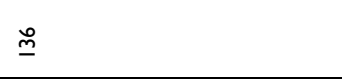 & $\hat{f}$ & & $\hat{n}$ & $\stackrel{\circ}{\circ}$ \\
\hline$\Omega^{\prime} \frac{\varrho}{\frac{\varrho}{\tilde{s}}}$ & $\stackrel{\circ}{\circ}$ & & & 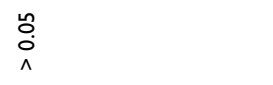 & \\
\hline 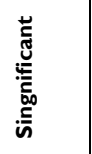 & $\stackrel{\Xi}{\varpi}$ & zo & 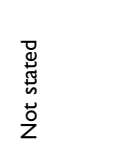 & $\stackrel{\circ}{z}$ & 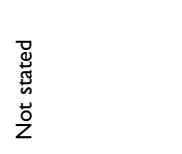 \\
\hline 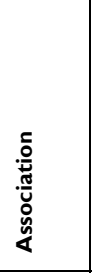 & 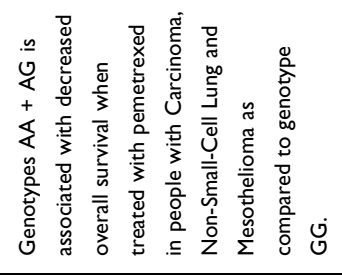 & 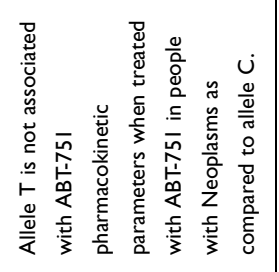 & 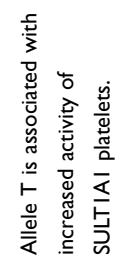 & 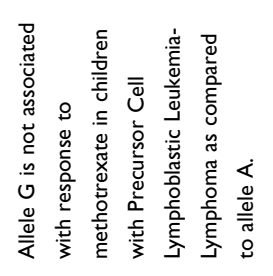 & 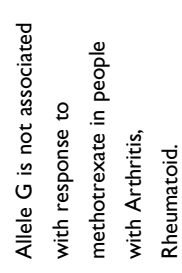 \\
\hline $\begin{array}{l}\frac{\tilde{\omega}}{\tilde{\Xi}} \\
\frac{\tilde{\omega}}{\Sigma} \\
\frac{\delta}{\Sigma}\end{array}$ & 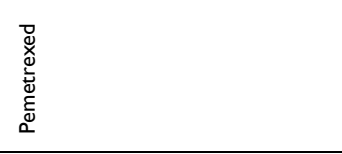 & 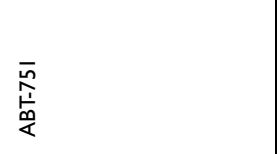 & & 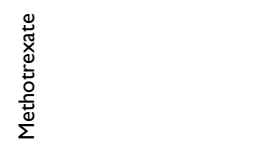 & 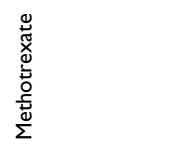 \\
\hline$\frac{\rho}{\sum_{\alpha}}$ & 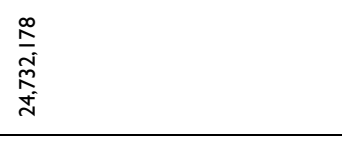 & 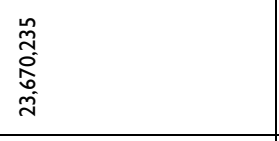 & 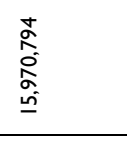 & 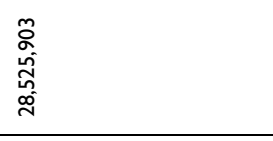 & 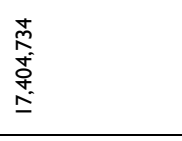 \\
\hline 蒿 & $\begin{array}{l}\frac{\infty}{\stackrel{\overbrace{}}{n}} \\
\frac{\hat{\alpha}}{\underline{n}}\end{array}$ & 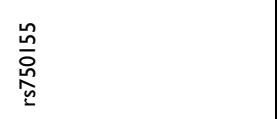 & 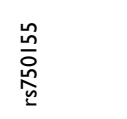 & $\frac{\stackrel{\circ}{\frac{\rho}{m}}}{\frac{m}{\underline{n}}}$ & $\frac{\stackrel{\circ}{\frac{h}{m}}}{\frac{m}{\underline{n}}}$ \\
\hline
\end{tabular}




\begin{tabular}{|c|c|c|}
\hline 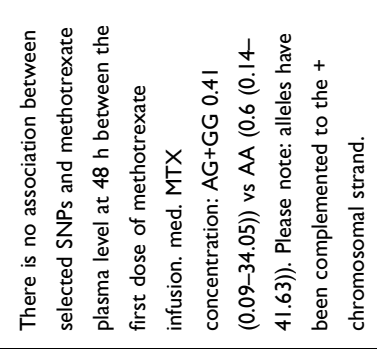 & & 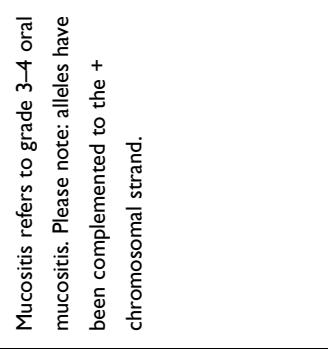 \\
\hline $\begin{array}{l}\overline{\bar{\alpha}} \\
\bar{u} \\
\bar{u}\end{array}$ & 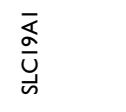 & $\begin{array}{l}\bar{\sigma} \\
\bar{U} \\
\bar{\omega}\end{array}$ \\
\hline 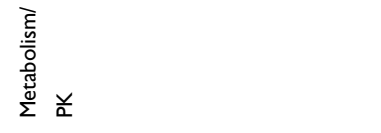 & & $\begin{array}{l}\stackrel{\vec{z}}{\vec{x}} \\
\stackrel{\circ}{\circ}\end{array}$ \\
\hline 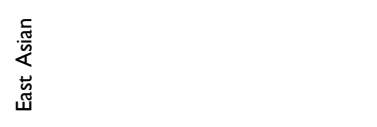 & & 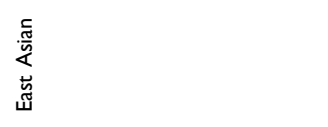 \\
\hline$\frac{1}{m}$ & & $\frac{\hat{m}}{m}$ \\
\hline$\frac{\hat{m}}{m}$ & & $\frac{\hat{m}}{m}$ \\
\hline ָे̀ & & $\begin{array}{l}\widehat{\hat{\sigma}} \\
0\end{array}$ \\
\hline$\stackrel{\circ}{z}$ & 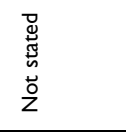 & o \\
\hline 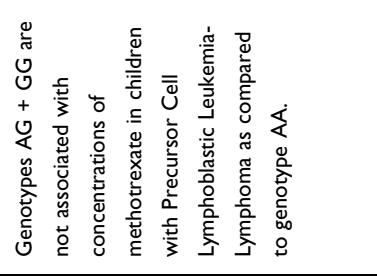 & 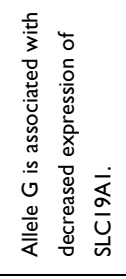 & 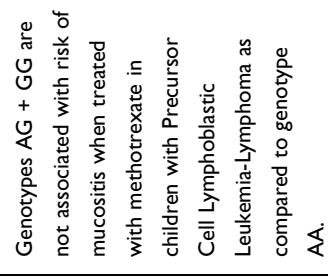 \\
\hline 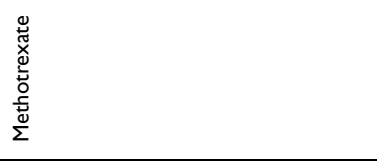 & & 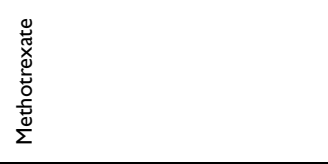 \\
\hline 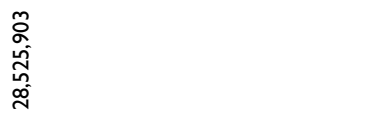 & 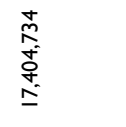 & 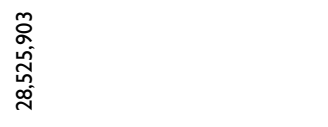 \\
\hline$\frac{\stackrel{\circ}{\circ}}{\frac{\stackrel{0}{m}}{m}}$ & $\begin{array}{l}\frac{\mathscr{0}}{\frac{h}{m}} \\
\frac{m}{\underline{n}}\end{array}$ & $\frac{\stackrel{\circ}{\frac{0}{m}}}{\bar{m}}$ \\
\hline
\end{tabular}


ratio. This study suggested that $[\mathrm{DHCQ}] /[\mathrm{HCQ}]$ ratio was associated with CYP2D6 rs1065852 polymorphism in lupus patients receiving oral HCQ. The CYP polymorphism may explain why the HCQ concentration in blood varies greatly. The effect of individual CYP polymorphisms should be considered in oral HCQ. Recently, LópezGarcía et $\mathrm{al}^{24}$ found single nucleotide polymorphisms associated with pharmacogenomics include CYP2D6*4 (rs1065852), which may affect the efficacy of antiepileptic drugs. Dlouhá et al reported no significant difference in the genotype/allelic frequencies of CYP2D6 (rs1065852) in Roma/Gypsy and Czech (non-Roma) populations. ${ }^{25}$ However, Zhang et al observed the frequency of CYP2D6 rs1065852 in the Lisu population different from the other populations. ${ }^{26}$ In our study, rs1065852-A frequency was the highest in the South East Asian populations. Therefore, to evaluate the effect of CYP2D6 (rs1065852) on drug metabolism, we should not ignore the ethnic factors, especially in the Asian populations.

The membrane protein Folate carrier protein 1 is involved in the regulation of intracellular folate concentration, and is encoded by SLC19A1 (Solute Carrier Family 19 Member 1) gene. $^{27}$ The mutant allele of SLC19A1 $-43 \mathrm{~T}>\mathrm{C}$ was reported to be associated with low folate levels. ${ }^{28}$ At present, the treatment response to pemetrexed has been proven to be individual specific. Zhang et al investigated the genetic characteristics of pemetrexed response in 203 Han patients with advanced non-small cell lung cancer (NSCLC). ${ }^{29}$ The participants who received pemetrexed alone, the SNP rs1051298 of SLC19A1 gene increased the risk of all adverse reactions in different cycles of the treatment. Therefore, rs 1051298 may be a marker associated with adverse reactions and efficacy of pemetrexed related therapy in Chinese Han patients. In addition, some researchers have found that rs1051296 was also related to drug reactions. Wang et al explored the influence of the miRNA binding site polymorphism (rs1051296) in SLC19A1 on the serum methotrexate (MTX) concentration in children with acute lymphoblastic leukaemia (ALL) after receiving MTX treatment. ${ }^{30}$ In comparison with the GT and TT carriers, the MTX concentration in GG carriers was higher than the treatment threshold. Compared with GT and TT carriers, the delayed elimination of MTX was observed in the GG carriers. Rs1051296 G>T correlated with the MTX plasma concentration. In the research, the frequency of rs 1051296 in Tibetans differed from that in the super-populations, leading to different genotypes of carriers such as MTX, for some drug efficacy.

The results will improve the pharmacogenomic information of the Tibetan population, and deepen the study on the differences in some genetic polymorphisms between the Tibetan population and the other 26 populations in the world. Some limitations of this study are: a small sample size and lack of experiment verification. Further studies are required on the drug efficacy of drug-related gene polymorphism loci in the Tibetan patients, especially for significant loci.

\section{Conclusions}

In conclusion, $A C E$ rs4291, SLC19A1 rs1051296 and CYP2D6 rs1065852 were significant in Tibetans compared to the other 26 nationalities. This study supplemented the knowledge of Tibetan pharmacology, and also showed the relationship between the SNPs with significant differences and drugs more perfectly, thus making the clinical medication safer and personalized for the Tibetan population.

\section{Data Sharing Statement}

The datasets used or analysed during the current study are available from the corresponding author upon reasonable request.

\section{Ethical Approval and Consent to Participate}

This study was performed in accordance with the World Medical Association Declaration of Helsinki and was approved by the Ethics Committee of the Affiliated Hospital of Xizang Minzu University. Written informed consent was obtained from all of the subjects before participation.

\section{Consent for Publication}

The authors have declared that they agreed to publish.

\section{Acknowledgment}

We are grateful to the participants for providing blood samples and extend our thanks to the professionals responsible for blood collection. We also thank the reviewers and editors for their patience.

\section{Funding}

The study was supported by Major Science and Technology Research Projects of Xizang (Tibet) 
Autonomous Region (2015XZ01G23), the Talent Development Supporting Project entitled Tibet-Shaanxi Himalaya of Xizang Minzu University (2020 Plateau Scholar), and Natural Science Foundation of Tibet Autonomous Region (2015ZR-13-19).

\section{Disclosure}

The authors report no conflicts of interest in this work.

\section{References}

1. Ma Q, Lu AY. Pharmacogenetics, pharmacogenomics, and individualized medicine. Pharmacol Rev. 2011;63(2):437-459. doi:10.1124/ pr.110.003533

2. Wang L, Aikemu A, Yibulayin A, et al. Genetic polymorphisms of pharmacogenomic VIP variants in the Uygur population from northwestern China. BMC Genet. 2015;16(1):66. doi:10.1186/s12863-0150232-x

3. Relling MV, Evans WE. Pharmacogenomics in the clinic. Nature. 2015;526(7573):343-350. doi:10.1038/nature15817

4. Jin T, Zhao R, Shi X, et al. Genetic polymorphisms study of pharmacogenomic VIP variants in Han ethnic of China's Shaanxi province. Environ Toxicol Pharmacol. 2016;46:27-35. doi:10.1016/j. etap.2016.06.026

5. Evans WE, McLeod HL. Pharmacogenomics-drug disposition, drug targets, and side effects. $N$ Engl J Med. 2003;348(6):538-549. doi:10.1056/NEJMra020526

6. Cheng Y, Dai R, Chen W, Li Q, Zhang C, Yang T. Genetic polymorphisms of pharmacogenomic VIP variants in the Dai population from Yunnan province. Mol Genet Genomic Med. 2020;8(7):e1231. doi:10.1002/mgg3.1231

7. Wang J, Chen Q, Wang L, et al. Identifying novel mutations of NKX2-5 congenital heart disease patients of Chinese minority groups. Int $J$ Cardiol. 2011;148(1):102-104. doi:10.1016/j. ijcard.2010.05.041

8. Yi X, Liang Y, Huerta-Sanchez E, et al. Sequencing of 50 human exomes reveals adaptation to high altitude. Science. 2010;329 (5987):75-78. doi:10.1126/science.1190371

9. Gabriel S, Ziaugra L, Tabbaa D. SNP genotyping using the Sequenom MassARRAY iPLEX platform. Curr Protocol Hum Gene. 2009;60(1):12. doi:10.1002/0471142905.hg0212s60

10. He Y, Yang H, Geng T, et al. Genetic polymorphisms of pharmacogenomic VIP variants in the lhoba population of southwest China. Int J Clin Exp Pathol. 2015;8(10):13293-13303.

11. Jin T, Yang H, Zhang J, et al. Polymorphisms and phenotypic analysis of cytochrome P450 3A4 in the Uygur population in northwest China. Int J Clin Exp Pathol. 2015;8(6):7083-7091.

12. Richard A. Gibbs, John W Belmont, Paul Hardenbol, et al. The International HapMap Project. Nature. 2003;426(6968):789-796. doi:10.1038/nature02168

13. Song MK, Lin FC, Ward SE, Fine JP. Composite variables: when and how. Nurs Res. 2013;62(1):45-49. doi:10.1097/NNR.0b013e3 182741948

14. Burrell LM, Harrap SB, Velkoska E, Patel SK. The ACE2 gene: its potential as a functional candidate for cardiovascular disease. Clin Sci. 2013;124(2):65-76. doi:10.1042/cs20120269

15. Probstfield JL, O'Brien KD. Progression of cardiovascular damage: the role of renin-angiotensin system blockade. Am $J$ Cardiol 2010;105(1Suppl):10a-20a. doi:10.1016/j.amjcard.2009.10.006
16. Jiang F, Yang J, Zhang Y, et al. Angiotensin-converting enzyme 2 and angiotensin 1-7: novel therapeutic targets. Nat Rev Cardiol. 2014;11 (7):413-426. doi:10.1038/nrcardio.2014.59

17. Magrone T, Magrone M, Jirillo E. Focus on receptors for coronaviruses with special reference to angiotensin- Converting enzyme 2 as a potential drug target - A perspective. Endocr Metab Immune Disord Drug Targets. 2020;20(6):807-811. doi:10.2174/ 1871530320666200427112902

18. Zhang Q, Cong M, Wang N, et al. Association of angiotensin-converting enzyme 2 gene polymorphism and enzymatic activity with essential hypertension in different gender: a case-control study. Medicine. 2018;97(42):e12917. doi:10.1097/md.0000000000012917

19. Fan Z, Wu G, Yue M, et al. Hypertension and hypertensive left ventricular hypertrophy are associated with ACE2 genetic polymorphism. Life Sci. 2019;225:39-45. doi:10.1016/j.lfs.2019.03.059

20. Martínez-Rodríguez N, Posadas-Romero C, Villarreal-Molina T, et al. Single nucleotide polymorphisms of the angiotensin-converting enzyme (ACE) gene are associated with essential hypertension and increased ACE enzyme levels in Mexican individuals. PLoS One. 2013;8(5):e65700. doi:10.1371/journal.pone.0065700

21. de Oliveira FF, Chen ES, Smith MC, Bertolucci PHF. Pharmacogenetics of angiotensin-converting enzyme inhibitors in patients with Alzheimer's disease dementia. Curr Alzheimer Res. 2018;15(4):386-398. doi:10.2174/1567205014666171016101816

22. Ferreira de Oliveira F, Berretta JM, Suchi CE, Cardoso SM, Ferreira BPH. Pharmacogenetic effects of angiotensin-converting enzyme inhibitors over age-related urea and creatinine variations in patients with dementia due to Alzheimer disease. Colomb Med. 2016;47 (2):76-80. doi: $10.25100 / \mathrm{cm} . v 47 i 2.2188$

23. Lee JY, Vinayagamoorthy N, Han K, et al. Association of polymorphisms of cytochrome P450 2D6 with blood hydroxychloroquine levels in patients with systemic lupus erythematosus. Arthritis Rheumatol. 2016;68(1):184-190. doi:10.1002/art.39402

24. López-García MA, Feria-Romero IA, Serrano H, et al. Influence of genetic variants of CYP2D6, CYP2C9, CYP2C19 and CYP3A4 on antiepileptic drug metabolism in pediatric patients with refractory epilepsy. Pharmacol Rep. 2017;69(3):504-511. doi:10.1016/j.pharep.2017.01.007

25. Dlouhá L, Adámková V, Šedová L, Olišarová V, Hubáček JA, Tóthová V. Five genetic polymorphisms of cytochrome P450 enzymes in the Czech non-Roma and Czech Roma population samples. Drug Metab Pers Ther. 2020. doi:10.1515/dmdi-2020-0103

26. Zhang C, Jiang X, Chen W, et al. Population genetic difference of pharmacogenomic VIP gene variants in the Lisu population from Yunnan Province. Medicine. 2018;97(52):e13674. doi:10.1097/ md.0000000000013674

27. Yee SW, Gong L, Badagnani I, Giacomini KM, Klein TE, Altman RB. SLC19A1 pharmacogenomics summary. Pharmacogenet Genomics. 2010;20(11):708-715. doi:10.107/FPC.0b013e32833eca92

28. Chatzikyriakidou A, Vakalis KV, Kolaitis N, et al. Distinct association of SLC19A1 polymorphism $-43 \mathrm{~T}>\mathrm{C}$ with red cell folate levels and of MTHFR polymorphism $677 \mathrm{C}>\mathrm{T}$ with plasma folate levels. Clin Biochem. 2008;41(3):174-176. doi:10.1016/j.clinbiochem.2007.11.006

29. Zhang X, Zhang D, Huang L, et al. Discovery of novel biomarkers of therapeutic responses in han chinese pemetrexed-based treated advanced NSCLC patients. Front Pharmacol. 2019;10:944. doi:10.3389/fphar.2019.00944

30. Wang S-M, Sun L-L, Zeng W-X, Wu W-S, Zhang G-L. Effects of a microRNA binding site polymorphism in SLC19A1 on methotrexate concentrations in Chinese children with acute lymphoblastic leukemia. Med Oncol. 2014;31(7):62. doi:10.1007/s12032-014-0062-0 


\section{Publish your work in this journal}

Pharmacogenomics and Personalized Medicine is an international, peer-reviewed, open access journal characterizing the influence of genotype on pharmacology leading to the development of personalized treatment programs and individualized drug selection for improved safety, efficacy and sustainability. This journal is indexed on the American Chemical Society's Chemical Abstracts Service (CAS). The manuscript management system is completely online and includes a very quick and fair peer-review system, which is al easy to use. Visit http://www.dovepress.com/testimonials.php to read real quotes from published authors. 